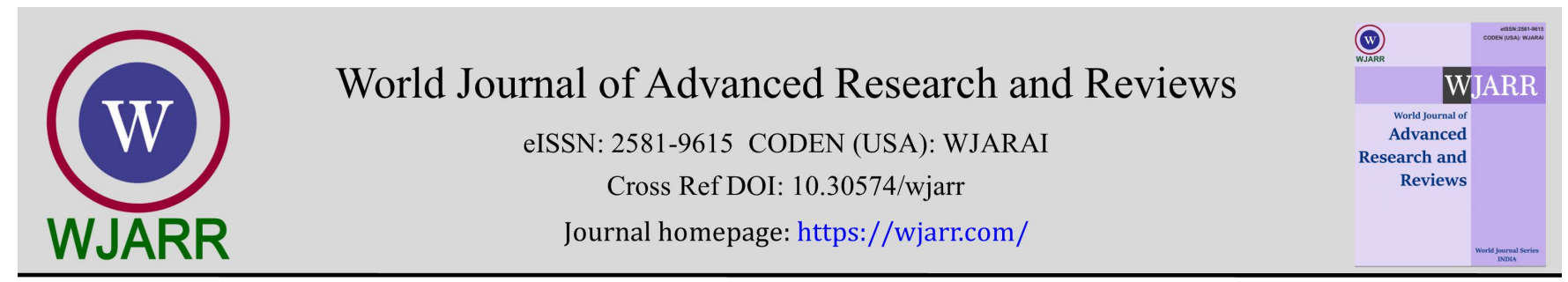

(REVIEW ARTICLE)

\title{
On class (BD) Operators
}

\author{
Wanjala Victor * and Beatrice Adhiambo Obiero \\ Department of Mathematics and computing, Rongo University, Kitere Hills Kenya.
}

World Journal of Advanced Research and Reviews, 2021, 11(02), 048-052

Publication history: Received on 26 June 2021; revised on 02 August 2021; accepted on 05 August 2021

Article DOI: https://doi.org/10.30574/wjarr.2021.11.2.0355

\begin{abstract}
In this paper, we introduce the class of (BD) operators acting on a complex Hilbert space $\mathrm{H}$. An operator if $\mathrm{T} \in \mathrm{B}(\mathrm{H})$ is said to belong to class (BD) if $\mathrm{T} * 2$ (TD) 2 commutes with (T *TD) 2 equivalently [T * 2 (TD) 2, (T *TD) 2] = 0. We investigate the properties of this class and we also analyze the relation of this class to D-operator and then generalize it to class (nBD) and analyze its relation to the class of n-power D-operator through complex symmetric operators.
\end{abstract}

Keywords: D-operator; Normal; N Quasi D-operator; Complex symmetric operators; N-power D-operator; (BD) Operators

\section{Introduction}

Throughout this paper, $\mathrm{H}$ denotes the usual Hilbert space over the complex field and B (H) the Banach algebra of all bounded linear algebra on an infinite dimensional separable Hilbert space $\mathrm{H}$. A bounded linear operator $\mathrm{T}$ is said to be in class $(\mathrm{Q})$ if $\mathrm{T}^{* 2} \mathrm{~T}^{2}=(\mathrm{T} * \mathrm{~T})^{2}(2)$. This was later extended into other classes like class $(\mathrm{Q})(2), \mathrm{n}$-power class (Q) if $\mathrm{T} *$ ${ }^{2} \mathrm{~T}^{2 \mathrm{n}}=\left(\mathrm{T}^{*} \mathrm{~T}^{\mathrm{n}}\right)^{2}(3)$, quasi-M class $(\mathrm{Q})$ and $(\alpha, \beta)$-class $(\mathrm{Q})$ we refer the reader to $(6)$ for more. An operator $\mathrm{T} \in \mathrm{B}(\mathrm{H})$ is said to belong to class (BQ) if $\mathrm{T}^{* 2} \mathrm{~T}^{2}(\mathrm{~T} * \mathrm{~T})^{2}=(\mathrm{T} * \mathrm{~T}){ }^{2} \mathrm{~T}^{* 2} \mathrm{~T}^{2}$ an operator $\mathrm{T} \in \mathrm{B}(\mathrm{H})$ is said to be $\mathrm{D}$-operator if $\mathrm{T}^{2}\left(\mathrm{~T}^{\mathrm{D}}\right){ }^{2}=$ $\left(\mathrm{T}{ }^{2} \mathrm{~T}^{\mathrm{D}}\right)^{2}$ where $\mathrm{T}^{\mathrm{D}}$ is the Drazin inverse of $\mathrm{T}(1)$. Wanjala Victor and A.M. Nyongesa later extended this to N Quasi Doperator (3), a bounded linear operator $\mathrm{T}$ is said to be $\mathrm{N}$ Quasi D-operator if $\mathrm{T}\left(\mathrm{T}^{2}\left(\mathrm{~T}^{\mathrm{D}}\right)^{2}\right)=\mathrm{N}\left(\mathrm{T}^{2} \mathrm{~T}^{\mathrm{D}}\right){ }^{2} \mathrm{~T}$ where $\mathrm{N}$ is a bounded linear operator. A bounded linear operator $\mathrm{T}$ is said to belong to class (BD) provided $\mathrm{T}^{2}\left(\mathrm{~T}^{\mathrm{D}}\right)^{2} \mathrm{commutes} \mathrm{with}^{2}$ $\left(\mathrm{T}^{2} \mathrm{~T}^{\mathrm{D}}\right)^{2}$ where $\mathrm{T}^{\mathrm{D}}$ is the Drazin inverse of $\mathrm{T}$. Let $\mathrm{H}$ be a Hilbert space, then a conjugation on $\mathrm{H}$ is an anti-linear operator $\mathrm{C}$ from $\mathrm{H}$ onto itself such that the following is satisfied $\mathrm{C} \xi, \mathrm{C} \zeta \mathrm{i}=\mathrm{h} \zeta, \xi i$ for every $\xi, \zeta \in \mathrm{H}$ and $\mathrm{C} 2=\mathrm{I}$. We say that $\mathrm{T}$ is complex symmetric if $\mathrm{T}=\mathrm{CT}{ }^{*} \mathrm{C}$.

\section{Main results}

\subsection{Theorem 1}

Let $\mathrm{T} \in \mathrm{B}(\mathrm{H})$ be such that $\mathrm{T} \in(\mathrm{BD})$, then the following are also true for (BD)

(i). $\lambda \mathrm{T}$ for any real $\lambda$

(ii). Any $\mathrm{S} \in \mathrm{B}(\mathrm{H})$ that is unitarily equivalent to $\mathrm{T}$.

(iii). the restriction T/ $\mathrm{M}$ to any closed subspace $\mathrm{M}$ of $\mathrm{H}$.

Proof. (i). the proof is trivial.

\footnotetext{
* Corresponding author: Wanjala Victor

Department of Mathematics and computing, Rongo University, Kitere Hills Kenya.

Copyright (@) 2021 Author(s) retain the copyright of this article. This article is published under the terms of the Creative Commons Attribution Liscense 4.0
} 
(ii). Let $\mathrm{S} \in \mathrm{B}(\mathrm{H})$ be unitarily equivalent to $\mathrm{T}$, then there exists a unitary operator $\mathrm{U}$

$\in \mathrm{B}(\mathrm{H})$ with

$\mathrm{S}=\mathrm{U} * \mathrm{TU}$ and $\mathrm{S}^{*}=\mathrm{U} * \mathrm{~T} * \mathrm{U}$. Since $\mathrm{T} \in(\mathrm{BD})$, we have;

$\mathrm{T}^{* 2}\left(\mathrm{~T}^{\mathrm{D}}\right)^{2}\left(\mathrm{~T}{ }^{*} \mathrm{~T}^{\mathrm{D}}\right)^{2}=\left(\mathrm{T}^{*} \mathrm{~T}^{\mathrm{D}}\right)^{2} \mathrm{~T}^{* 2}\left(\mathrm{~T}^{\mathrm{D}}\right)^{2}$, hence

$\mathrm{S}^{* 2}\left(\mathrm{~S}^{\mathrm{D}}\right)^{2}\left(\mathrm{~S}^{*} \mathrm{~S}^{\mathrm{D}}\right)^{2}=\mathrm{UT}{ }^{*}{ }^{2} \mathrm{U} * \mathrm{U}\left(\mathrm{T}^{\mathrm{D}}\right)^{2} \mathrm{U} *\left(\mathrm{UT} * \mathrm{U} * \mathrm{UT}{ }^{\mathrm{D} U} \mathrm{U}^{*}{ }^{2}\right.$

$=\mathrm{UT} *{ }^{2} \mathrm{U} * \mathrm{U} *\left(\mathrm{~T}^{\mathrm{D}}\right){ }^{2} \mathrm{U} * \mathrm{UT} * \mathrm{U} * \mathrm{UT} * \mathrm{U} * \mathrm{UT}{ }^{\mathrm{D} U} * \mathrm{UT}{ }^{\mathrm{D}} \mathrm{U} *$

$=\mathrm{UT} * 2\left(\mathrm{~T}^{\mathrm{D}}\right)^{2}\left(\mathrm{~T}^{*} \mathrm{~T}^{\mathrm{D}}\right){ }^{2} \mathrm{U} *$

$=\mathrm{U}\left(\mathrm{T} * \mathrm{~T}^{\mathrm{D}}\right){ }^{2} \mathrm{~T}^{* 2}\left(\mathrm{~T}^{\mathrm{D}}\right)^{2} \mathrm{U} *$

And

$\left(S^{*} S^{D}\right){ }^{2} S^{* 2}\left(S^{D}\right){ }^{2}=\left(U T{ }^{*} U * U T\right.$ DU * ${ }^{2} U T{ }^{2}{ }^{2}{ }^{*} U\left(T^{D}\right)^{2} U$ *

$=\mathrm{UT} * \mathrm{U} * \mathrm{U}\left(\mathrm{T}{ }^{\mathrm{D}}\right) \mathrm{U} * \mathrm{UT} * \mathrm{U} * \mathrm{U}$ T ${ }^{\mathrm{D}} \mathrm{U} * \mathrm{UT} *{ }^{2} \mathrm{U} * \mathrm{U}\left(\mathrm{T}{ }^{\mathrm{D}}\right){ }^{2} \mathrm{U} *$

$=\mathrm{UT} * \mathrm{~T}{ }^{\mathrm{D}} \mathrm{T} * \mathrm{~T}{ }^{\mathrm{D}} \mathrm{T} *{ }^{2} \mathrm{~T} * 2 \mathrm{U} *$

$=\mathrm{U}\left(\mathrm{T} * \mathrm{~T}^{\mathrm{D}}\right){ }^{2} \mathrm{~T}^{* 2}\left(\mathrm{~T}{ }^{\mathrm{D}}\right){ }^{2} \mathrm{U} *$

Hence $\mathrm{S}$ is unitarily equivalent to $\mathrm{T}$.

(iii). If $\mathrm{T}$ is in class (BD), then;

$\mathrm{T}^{* 2}\left(\mathrm{~T}^{\mathrm{D}}\right)^{2}\left(\mathrm{~T}^{*} \mathrm{~T}^{\mathrm{D}}\right)^{2}=\left(\mathrm{T}^{*} \mathrm{~T}^{\mathrm{D}}\right)^{2} \mathrm{~T}^{* 2}\left(\mathrm{~T}^{\mathrm{D}}\right)^{2}$.

Hence;

$(\mathrm{T} / \mathrm{M}) *{ }^{2}\left((\mathrm{~T} / \mathrm{M}){ }^{\mathrm{D}}\right)^{2}\left\{(\mathrm{~T} / \mathrm{M}) *(\mathrm{~T} / \mathrm{M}){ }^{\mathrm{D}}\right\}^{2}$

$=(\mathrm{T} / \mathrm{M}) * 2\left((\mathrm{~T} / \mathrm{M}){ }^{\mathrm{D}}\right)^{2}\left\{(\mathrm{~T} / \mathrm{M}) *(\mathrm{~T} / \mathrm{M}){ }^{\mathrm{D}}\right\}^{2}$

$=(\mathrm{T} * 2 / \mathrm{M})\left(\left(\mathrm{T}^{\mathrm{D}}\right)^{2} / \mathrm{M}\right)\left\{(\mathrm{T} * / \mathrm{M})\left(\mathrm{T}^{\mathrm{D}} / \mathrm{M}\right)\right\}\left\{(\mathrm{T} * / \mathrm{M})\left(\mathrm{T}^{\mathrm{D}} / \mathrm{M}\right)\right\}$

$=\left\{\left(\mathrm{T}^{*} \mathrm{~T}^{\mathrm{D}}\right)^{2} / \mathrm{M}\right\}\left\{\mathrm{T}{ }^{* 2}\left(\mathrm{~T}^{\mathrm{D}}\right)^{2} / \mathrm{M}\right\}$

$=\left\{(\mathrm{T} * / \mathrm{M})\left(\mathrm{T}^{\mathrm{D}} / \mathrm{M}\right)\right\}^{2}(\mathrm{~T} / \mathrm{M}) * 2\left((\mathrm{~T} / \mathrm{M}){ }^{\mathrm{D}}\right)^{2}$

Hence $\mathrm{T} / \mathrm{M} \in(\mathrm{BD})$.

\subsection{Theorem 2}

If $T \in B(H)$ is a D-operator, then $T \in(B D)$.

Proof. Suppose T is a D-operator, then

$\mathrm{T} * 2\left(\mathrm{~T}^{\mathrm{D}}\right)^{2}=(\mathrm{T} * \mathrm{~T})^{2}$

Post multiplying both sides by $\mathrm{T}^{*}{ }^{2}\left(\mathrm{~T}^{\mathrm{D}}\right)^{2}$;

$\mathrm{T}^{* 2}\left(\mathrm{~T}^{\mathrm{D}}\right)^{2} \mathrm{~T}^{* 2}\left(\mathrm{~T}^{\mathrm{D}}\right)^{2}=\left(\mathrm{T}^{*} \mathrm{~T}^{\mathrm{D}}\right)^{2} \mathrm{~T}^{* 2}\left(\mathrm{~T}^{\mathrm{D}}\right)^{2}$

$\mathrm{T} * 2\left(\mathrm{~T}^{\mathrm{D}}\right)^{2} \mathrm{~T} * \mathrm{~T}$ D $\mathrm{T} * \mathrm{~T} \mathrm{D}=(\mathrm{T} * \mathrm{TD}) 2 \mathrm{~T} * 2(\mathrm{~T})^{2}$ 
$\mathrm{T}^{* 2}\left(\mathrm{~T}^{\mathrm{D}}\right)^{2}\left(\mathrm{~T}^{*} \mathrm{~T}^{\mathrm{D}}\right)^{2}=\left(\mathrm{T}^{*} \mathrm{~T}^{\mathrm{D}}\right)^{2} \mathrm{~T}^{* 2}\left(\mathrm{~T}^{\mathrm{D}}\right)^{2}$.

\subsection{Theorem 3}

Let $S \in(B D)$ and $T \in(B D)$. If both $S$ and $T$ are doubly commuting, then

$\mathrm{ST}$ is in (BD).

Proof.

$(\mathrm{ST})^{* 2}\left((\mathrm{ST})^{\mathrm{D}}\right)^{2}\left((\mathrm{ST}) *(\mathrm{ST})^{\mathrm{D}}\right)^{2}$

$\left.=\mathrm{S}^{* 2} \mathrm{~T}^{* 2}\left(\mathrm{~S}^{\mathrm{D}}\right)^{2}\left(\mathrm{~T}^{\mathrm{D}}\right)^{2}\left((\mathrm{ST}) *(\mathrm{ST}){ }^{\mathrm{D}}\right)((\mathrm{ST}) *(\mathrm{ST}))^{\mathrm{D}}\right)$

$=\mathrm{S}^{* 2} \mathrm{~T}^{* 2}\left(\mathrm{~S}^{\mathrm{D}}\right)^{2}\left(\mathrm{~T}^{\mathrm{D}}\right)^{2}\left(\left(\mathrm{~S}^{*} \mathrm{~T} *\right)(\mathrm{ST})^{\mathrm{D}}\right)\left(\left(\mathrm{S}^{*} \mathrm{~T} *\right)(\mathrm{ST})^{\mathrm{D}}\right)$

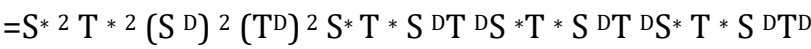

$=\mathrm{S} *{ }^{2} \mathrm{~T} *{ }^{2}(\mathrm{SD})^{2}(\mathrm{TD})^{2} \mathrm{~S} *(\mathrm{SD}) \mathrm{T} *(\mathrm{~T} \mathrm{D}) \mathrm{S} *(\mathrm{SD}) \mathrm{T} * \mathrm{TD}$

$=\mathrm{T}^{* 2}\left(\mathrm{~T}^{\mathrm{D}}\right)^{2} \mathrm{~S}^{* 2}\left(\mathrm{~S}^{\mathrm{D}}\right)^{2} \mathrm{~S}^{*} \mathrm{~S}$ DS* $\mathrm{S}$ $\mathrm{T} * \mathrm{~T}$ DT $* \mathrm{~T}^{\mathrm{D}}$

$=\mathrm{T}^{* 2}\left(\mathrm{~T}^{\mathrm{D}}\right)^{2} \mathrm{~S}^{* 2}\left(\mathrm{~S}^{\mathrm{D}}\right)^{2}\left(\mathrm{~S}^{*} \mathrm{~S}^{\mathrm{D}}\right){ }^{2} \mathrm{~T} * \mathrm{~T} \mathrm{D}^{\mathrm{T}} \mathrm{T}^{\mathrm{D}}$

$=T^{* 2}\left(T^{D}\right){ }^{2}\left(S^{*} S^{D}\right){ }^{2} S^{* 2}\left(S^{D}\right)^{2} T^{*} T^{D T}{ }^{*} T^{D}($ Since $S \in(B D))$.

$=(\mathrm{S} * \mathrm{~S})^{2} \mathrm{~T} * 2\left(\mathrm{~T}^{\mathrm{D}}\right)^{2} \mathrm{~T} * \mathrm{~T} \mathrm{DT} * \mathrm{~T}$ DS $* 2(\mathrm{~S} \text { D })^{2}$

$=\left(\mathrm{S}^{*} \mathrm{~S}^{\mathrm{D}}\right)^{2} \mathrm{~T}^{* 2}\left(\mathrm{~T}^{\mathrm{D}}\right)^{2}\left(\mathrm{~T}^{*} \mathrm{~T}^{\mathrm{D}}\right)^{2} \mathrm{~S}^{* 2}\left(\mathrm{~S}^{\mathrm{D}}\right)^{2}$

$=\left(\mathrm{S}^{*} \mathrm{~S}^{\mathrm{D}}\right)^{2}\left(\mathrm{~T}^{*} \mathrm{~T}^{\mathrm{D}}\right)^{2} \mathrm{~T}^{* 2}\left(\mathrm{~T}^{\mathrm{D}}\right)^{2} \mathrm{~S}^{* 2}\left(\mathrm{~S}^{\mathrm{D}}\right)^{2}($ Since $\mathrm{T} \in(\mathrm{BD}))$.

$=\left(\left(\mathrm{S}^{*} \mathrm{~S}^{\mathrm{D}}\right)\left(\mathrm{T}^{*} \mathrm{~T}^{\mathrm{D}}\right)\right)^{2} \mathrm{~T}^{* 2} \mathrm{~S}^{* 2}\left(\mathrm{~T}^{\mathrm{D}}\right)^{2}\left(\mathrm{~S}^{\mathrm{D}}\right)^{2}$

$=\left(\left(\mathrm{S}^{*} \mathrm{~T}^{*}\right)\left(\mathrm{S}^{\mathrm{D}} \mathrm{T}^{\mathrm{D}}\right)\right)^{2} \mathrm{~S}^{* 2} \mathrm{~T}^{* 2}\left(\mathrm{~S}^{\mathrm{D}}\right)^{2}\left(\mathrm{~T}^{\mathrm{D}}\right)^{2}$

$\left.\left.=((\mathrm{ST}) *(\mathrm{ST}))^{\mathrm{D}}\right)^{2}(\mathrm{ST}) * 2((\mathrm{ST}))^{\mathrm{D}}\right)^{2}$

Hence ST $\in(B D)$.

\subsection{Theorem 4}

Let $\mathrm{T} \in \mathrm{B}(\mathrm{H})$ be a class (BD) operator such that $\mathrm{T}=\mathrm{CT}{ }^{*} \mathrm{C}$ with $\mathrm{C}$ being a

Conjugation on $\mathrm{H}$. If $\mathrm{C}$ is such that it commutes with $\mathrm{T}^{* 2}\left(\mathrm{~T}^{\mathrm{D}}\right)^{2}$ and $\left(\mathrm{T}^{*} \mathrm{~T}^{\mathrm{D}}\right)^{2}$, then $\mathrm{T}$ is a

D- Operator.

Proof. Let $T \in(B D)$ and complex symmetric, then we have; $T^{* 2}\left(T^{\mathrm{D}}\right)^{2}\left(\mathrm{~T}^{*} \mathrm{~T}^{\mathrm{D}}\right)^{2}=\left(\mathrm{T}^{*} \mathrm{~T}^{\mathrm{D}}\right)^{2} \mathrm{~T}^{* 2}\left(\mathrm{~T}^{\mathrm{D}}\right)^{2}$

And $\mathrm{T}=\mathrm{CT} * \mathrm{C}$.

Hence;

$\mathrm{T}^{* 2}\left(\mathrm{~T}^{\mathrm{D}}\right)^{2}\left(\mathrm{~T} * \mathrm{~T}^{\mathrm{D}}\right)^{2}=\left(\mathrm{T} * \mathrm{~T}^{\mathrm{D}}\right)^{2} \mathrm{~T}^{* 2}\left(\mathrm{~T}^{\mathrm{D}}\right)^{2}$

$\mathrm{T}^{* 2}\left(\mathrm{~T}^{\mathrm{D}}\right)^{2} \mathrm{CT}$ DCCT $*$ CCT ${ }^{\mathrm{DCCT}} * \mathrm{C}=(\mathrm{T} * \mathrm{~T})^{2} \mathrm{CT}$ DCCT *CCT ${ }^{\mathrm{D} C C T} * \mathrm{C}$.

$\mathrm{T} * 2\left(\mathrm{~T}^{\mathrm{D}}\right)^{2} \mathrm{CT}$ D $\mathrm{T} * \mathrm{~T}{ }^{\mathrm{D}} \mathrm{T} * \mathrm{C}=\left(\mathrm{T} * \mathrm{~T}^{\mathrm{D}}\right){ }^{2} \mathrm{CT} \mathrm{DT}^{*} \mathrm{~T}{ }^{\mathrm{D}} \mathrm{T} * \mathrm{C}$ 
$\mathrm{T}^{* 2}\left(\mathrm{~T}^{\mathrm{D}}\right)^{2} \mathrm{C}\left(\mathrm{T}\right.$ D) ${ }^{2} \mathrm{~T} *{ }^{2} \mathrm{C}=\left(\mathrm{T} * \mathrm{TD}^{2}\right)^{2} \mathrm{CT} * \mathrm{~T}$ DT $* \mathrm{~T}$ D $\mathrm{C}$

$\mathrm{T}^{* 2}\left(\mathrm{~T}^{\mathrm{D}}\right)^{2} \mathrm{CT} * 2\left(\mathrm{~T}^{\mathrm{D}}\right)^{2} \mathrm{C}=\left(\mathrm{T} * \mathrm{~T}^{\mathrm{D}}\right)^{2} \mathrm{C}\left(\mathrm{T} * \mathrm{~T}^{\mathrm{D}}\right)^{2} \mathrm{C}$.

C commutes with $\mathrm{T}^{* 2}\left(\mathrm{~T}^{\mathrm{D}}\right)^{2}$ and $\left(\mathrm{T}^{*} \mathrm{~T}^{\mathrm{D}}\right)^{2}$ hence we obtain;

$\mathrm{T}^{* 2}\left(\mathrm{~T}^{\mathrm{D}}\right)^{2} \mathrm{~T}^{* 2}\left(\mathrm{~T}^{\mathrm{D}}\right)^{2}=\left(\mathrm{T} * \mathrm{~T}^{\mathrm{D}}\right)^{2}\left(\mathrm{~T} * \mathrm{~T}^{\mathrm{D}}\right)^{2}$.

Which implies;

$\mathrm{T}^{* 2}\left(\mathrm{~T}^{\mathrm{D}}\right)^{2}=\left(\mathrm{T}^{*} \mathrm{~T}^{\mathrm{D}}\right)^{2}$ and hence $\mathrm{T}$ is a D-operator.

Definition 5. An operator $\mathrm{T}$ is said to be in class $(\mathrm{nBD})$ if $\mathrm{T}^{* 2}\left(\mathrm{~T}^{\mathrm{D}}\right)^{2 \mathrm{n}}\left(\mathrm{T}^{*}\left(\mathrm{~T}^{\mathrm{D}}\right)^{\mathrm{n}}\right)^{2}=\left(\mathrm{T}^{*}\left(\mathrm{~T}^{\mathrm{D}}\right)^{\mathrm{n}}\right)^{2} \mathrm{~T}^{* 2}\left(\mathrm{~T}^{\mathrm{D}}\right)^{2 \mathrm{n}}$ for a positive integer $n$.

\subsection{Theorem 6}

Let $T \in B(H)$ be $(n-1)-D-$ operator, if $T$ is a complex symmetric

Operator such that $\mathrm{C}$ commutes with $\left(\mathrm{T}^{*} \mathrm{~T}^{\mathrm{D}}\right)^{2}$, then $\mathrm{T}$ is an $\mathrm{n}$-power $\mathrm{D}$ - operator.

Proof. With T being complex symmetric and (n-1)-D-operator, we have;

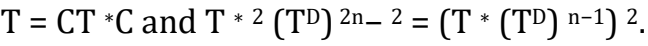

We obtain;

$\mathrm{T}^{* 2}\left(\mathrm{~T}^{\mathrm{D}}\right)^{2 \mathrm{n}}-2\left(\mathrm{~T}^{\mathrm{D}}\right)^{2}=\left(\mathrm{T}^{*}\left(\mathrm{~T}^{\mathrm{D}}\right)^{\mathrm{n}-1}\right)^{2}\left(\mathrm{~T}^{\mathrm{D}}\right)^{2}$

Hence;

$\mathrm{T}^{* 2}\left(\mathrm{~T}^{\mathrm{D}}\right)^{2 \mathrm{n}}=\left(\mathrm{T}^{*}\left(\mathrm{~T}^{\mathrm{D}}\right)^{\mathrm{n}-1}\right)^{2}\left(\mathrm{~T}^{\mathrm{D}}\right)^{2}$

$\mathrm{T}^{* 2}\left(\mathrm{~T}^{\mathrm{D}}\right)^{2 \mathrm{n}}=\mathrm{T}^{* 2}\left(\mathrm{~T}^{\mathrm{D}}\right)^{2 \mathrm{n}}-2\left(\mathrm{~T}^{\mathrm{D}}\right)^{2}=\left(\mathrm{T}^{\mathrm{D}}\right)^{2 \mathrm{n}}-2 \mathrm{~T}^{* 2}\left(\mathrm{~T}^{\mathrm{D}}\right)^{2}$

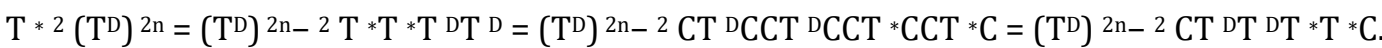

$=\mathrm{T}^{* 2}\left(\mathrm{~T}^{\mathrm{D}}\right)^{2 \mathrm{n}}=\left(\mathrm{T}^{\mathrm{D}}\right)^{2 \mathrm{n}}-{ }^{2} \mathrm{C}\left(\mathrm{T}^{\mathrm{D}}\right)^{2} \mathrm{~T}^{* 2} \mathrm{C}=\left(\mathrm{T}^{\mathrm{D}}\right)^{2 \mathrm{n}}-{ }^{2} \mathrm{C}\left(\mathrm{T}^{*} \mathrm{~T}^{\mathrm{D}}\right)^{2} \mathrm{C}$

Since C commutes with $\left(\mathrm{T} * \mathrm{~T}^{\mathrm{D}}\right)^{2}$ we obtain;

$\mathrm{T}^{* 2}\left(\mathrm{~T}^{\mathrm{D}}\right)^{2 \mathrm{n}}=\left(\mathrm{T}^{\mathrm{D}}\right)^{2 \mathrm{n}_{-} 2}\left(\mathrm{~T}^{*} \mathrm{~T}^{\mathrm{D}}\right)^{2} \mathrm{CC}=\left(\mathrm{T}^{\mathrm{D}}\right)^{2 \mathrm{n}_{-}}{ }^{2} \mathrm{~T}^{* 2}\left(\mathrm{~T}^{\mathrm{D}}\right)^{2} \mathrm{CC}=\left(\mathrm{T}^{\mathrm{D}}\right)^{2 \mathrm{n}_{-}}{ }^{2}\left(\mathrm{~T}^{\mathrm{D}}\right)^{2} \mathrm{~T}^{* 2} \mathrm{CC}=\mathrm{T}^{* 2}\left(\mathrm{~T}^{\mathrm{D}}\right)^{2 \mathrm{n}}=\left(\mathrm{T}^{*}\left(\mathrm{~T}^{\mathrm{D}}\right)^{\mathrm{G}}\right)^{2}$

Hence $\mathrm{T}$ is n-power D-operator

\section{Conclusion}

The study of class (BD) operators will help in the enhancement of study of properties of various classes such as class (Q) operators, normal operators and binormal operators.

\section{Compliance with ethical standards}

\section{Acknowledgments}

The researchers appreciated all the comments and inputs made by experts before publication.

\section{Disclosure of conflict of interest}

The authors declared no conflict of interest. 


\section{References}

[1] Abood, Kadhim. Some properties of D-operator, Iraqi Journal of Science. 2021; 61(12); 3366-3371.

[2] Jibril AAS. On Operators for which $\mathrm{T}^{* 2}(\mathrm{~T})^{2}=(\mathrm{T} * \mathrm{~T})^{2}$, international mathematical forum. 5 (46): 2255-2262.

[3] S Paramesh, D Hemalatha, VJ Nirmala. A study on n-power class (Q) operators, international research journal of engineering and technology. 2019; 6(1): 2395-0056.

[4] Wanjala Victor, AM Nyongesa. On N Quasi D-operators, international journal of mathematics and its applications. 2021; 9(2): 245-248.

[5] Wanjala Victor and Beatrice Adhiambo Obiero., On almost class $(\mathrm{Q})$ and class $(\mathrm{M}, \mathrm{n})$ operators, international journal of mathematics and its applications. 2021; 9(2): 115-118.

[6] Wanjala Victor, AM Nyongesa. On $(\alpha, \beta)$-class $(Q)$ Operators, international journal of mathematics and its applications. 2021; 9(2): 111-113. 